\title{
Altering Surface Topography of Electrospun Fibers
}

\author{
Murat Şimşek ${ }^{*} 1$ iD
}

\begin{abstract}
When considering fiber-based materials, electrospinning is a fascinating method for producing polymeric nano/micro fibers in comparison with other techniques. Its wide range of applications from engineering to medicine make electrospinning gain great interest. Beside fibrous structure of fiber mats, topographical features on and/or inside fiber surfaces make them ideal candidates for site-specific applications. Several approaches have been adopted to gain desired topographical textures on individual fiber surfaces. Solvent properties, environmental conditions and also conditions for preparing fibers induce topographical changes in various physical characteristics.

Keywords

Metal carbonyls; Surface Topography; Porous Fibers; Electrospinning; Humidity; Phase Separation.

${ }^{1}$ Inonu University, Biomedical Engineering, Malatya, Turkey

*Corresponding Author: murat.simsek@inonu.edu.tr

Manuscript received date: May 24, 2019

Accept Date: June 26, 2019

Published Date: June 30, 2019
\end{abstract}

\section{INTRODUCTION}

Electrospinning is a versatile technique that uses an external electric field to produce polymeric micro/nano sized fibers (Şimşek, 2018). Electrospinning includes the acceleration of a charged jet of polymer solution towards a grounded collector. The solvent evaporation and the stretching of the jet, caused by the repulsive forces of the charged molecules within the jet, are responsible for the formation of the polymer fibers. Electrospun micro and nanofibers have notable characteristics such as a large surface area to volume ratio, good pore interconnectivity, and high porosity. A further increase of the surface area by incorporating tailored surface topographies is therefore of major interest for many applications including tissue engineering, drug delivery, catalysis, filtration and sensors (Baker et al., 2015; Chen and Tung, 2017; D. Li and Xia, 2004; Liu et al., 2015; J. Yu et al., 2008). For example, porosity in electrospun polymer fibers has great advantageous for tissue engineering. The porous surface of nano or micro structure is not only important for an increase in cell attachment and tissue compatibility but also prerequisite for the transport of oxygen and nutrient supply to the cells and for the cellular growth. Porous nanofibers can be obtained by selecting particular solvents or solvent mixtures, polymer mixtures, optimizing electrospinning conditions and/or controlling environmental conditions.

In the presented review, several mechanisms involved in the production of electrospun fibers having various topographical textures are identified in the presence of literature.

\section{MECHANISMS}

A number of methods have been adopted to prepare electrospun nanofibers at various surface characteristics. The following subsections were organized as based on their mechanisms that induce surface textures on electrospun fibers in different manner.

\subsection{Breath figures}

The breath figures mechanism was firstly observed by Srinivasarao et al. (Srinivasarao, Collings, Philips, and Patel, 2001), who described the formation of pores on a polystyrene (PS) film in a humid environment. In that case the solvent evaporation causes a lowering of the temperature on the surface of the film. Water vapor from the atmosphere is condensed on the surface, the resulting water condensation begins with the nucleation of droplets in random positions followed by coalescence and potential organization to minimize thermodynamic energy (Marcos-Martin, Beysens, Bouchaud, Godrèche, and Yekutieli, 1995). Finally, the pores were created by the evaporation of the water molecules from the surface (Figure $1 \mathrm{a}$ ). The use of humidity to increase 
the porosity of electrospun fibers has also been proposed by other researchers (Casper, Stephens, Tassi, Chase, and Rabolt, 2004; Nezarati, Eifert, and Cosgriff-Hernandez, 2013).

Breath figures are nano-to micron- sized patterned arrays of defects. However, the pores formed on electrospun fibers are not uniform and this may be due to the dynamic condition of the electrospinning jet as compared to the static conditions where the effects of breath figures are dominant in thin films (Ramakrishna). In addition, this process is relatively slower compared with phase separation and only forms pores on the surface of electrospun nanofibers (Lu and Xia, 2013). Nevertheless, porous nanofibers can be obtained by electrospinning of a kind of hydrophobic polymer from a volatile and water-compatible solvent at a proper level of relative humidity.
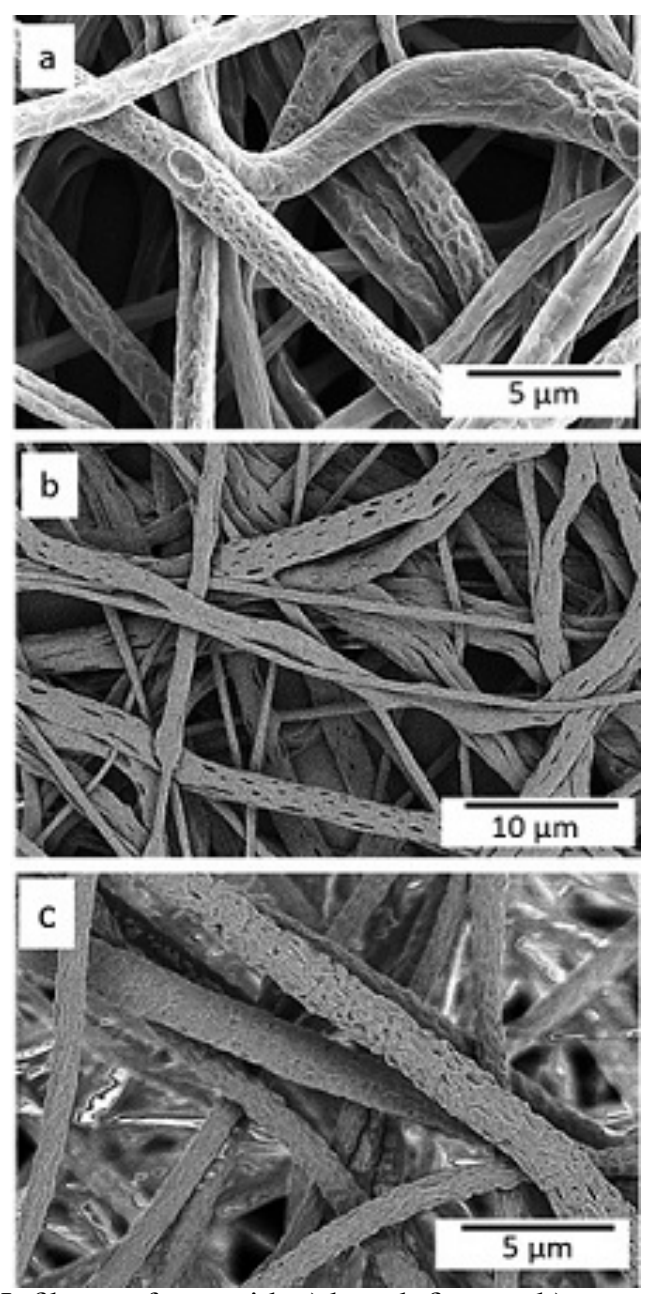

Figure 1. PCL fiber surfaces with a) breath figures, b) pores and c) rough

\subsection{Non-solvent induced phase separation (NIPS)}

There are two kinds of NIPS processes. The first one is also known as immersion precipitation method by which a cast polymer solution is immersed in a nonsolvent bath to initiate phase separation (D.-J. Lin, Chang, Chen, Lee, and Cheng, 2006). The other one is based on mixing solvent and non-solvent having higher boiling point than that of solvent with an appropriate amount that does not cause phase separation before electrospinning. In immersion precipitation, the polymer precipitates rapidly after immersion in a non-solvent bath resulting in highly porous structures. A simple and efficient method to induce porosity both in the core and on the surface of electrospun fibers were demonstrated (Nayani et al., 2012). Porous fibers were obtained for poly(acrylonitrile) (PAN)/dimethyl formamide (DMF), PS/DMF, PS/toluene, and poly(methyl methacrylate)/DMF polymer/solvent systems when using non-solvent water bath as collector. Authors concluded that water and DMF have excellent miscibility which favored the generation of porosity by NIPS. Apart from that nearly smooth fibers having no surface porosity were obtained when hexane was used as the nonsolvent bath. In the case where solvent and non-solvent are used, the composition changes and falls into the phase separation region because of the different volatility between solvent and nonsolvent.Thus, porous structures can be created during the subsequent solidification process of electrospinning (Katsogiannis, Vladisavljević, and Georgiadou, 2015; Lubasova and Martinova, 2011; Qi, Yu, Chen, and Zhu, 2009; Wei et al., 2013) (Figure 
$1 \mathrm{~b}$ ). The presence of a non-solvent can induce thermodynamic instability of the solution and initiates the phase separation process. An advantage of NIPS over the other phase separation mechanisms is that it can be controlled and can provide reproducible results.

PS fibers with micro- and nano-porous structures both in the core and/or on the fiber surfaces were electrospun by varying solvent compositions and solution concentrations of the PS solutions (J. Lin, Ding, and Yu, 2010). According to the authors the tetrahydrofuran (THF)/DMF mixing ratio in PS solutions was proved to be the key parameter to affect porous structure. In the process, the phase separation resulted from rapid evaporation of solvent in electrospinning. The high vapor pressure solvent THF created the fibers comprising nanoporous sheaths with 3D interconnected nanofibrils cores. As the vapor pressure of the solvent mixtures decreased, the micro and nanoporous structures on the fiber surfaces disappeared but wrinkled or smooth surfaces presented. The fibrous mats electrospun from 1/3 THF/DMF solvent system showed largest specific surface area and pore volume. In a study by Byong-Taek et al. (Nguyen, Bao, Park, and Lee, 2013), fibrous scaffolds composed of electrospun porous poly(e-caprolactone) (PCL) fibers were produced for bone tissue engineering. In this study, various ratios of dichloromethane (DCM)/Acetone (ACT) (10/0, 8/2, 6/4 and 5/5, v/v) solvents were used to dissolve PCL, which could affect the surface structure of the fibers. When only DCM was used, the morphology of the PCL fiber surface was rough. However, when DCM/ACT (50/50) solvent was used, the PCL fibers were more porous with many pores with size distribution in the range of 500-1000 nm. In vitro and in vivo results verified that porous PCL fiber-based fibrous scaffolds after $12 \mathrm{~h}$ of immersion in simulated body fluid was excellent for cell interaction, growth, proliferation and enabled greater acceleration of bone formation than other samples. According to a study by Tung and Chen (Chen and Tung, 2017), the pore size of PS fibers increased with increasing dimethyl sulfoxide (DMSO) fraction, indicating that the nonsolvent must play an important role in the formation of the pores. Moreover, the shape and orientation of the pores are affected by the stretching forces. The porous structure imparted superhydrophobic surface to the fibrous mats, and thus the fibers could selectively adsorb oils while repelled water. Georgiadou et al. (Katsogiannis et al., 2015) concluded that the pore formation was favored at high good/poor solvent ratios. In their study, the effect of the solvent properties on the size and surface morphology of electrospun PCL fibers was investigated. Chloroform (CF), DCM, THF and formic acid (FA) were used as good solvents in mixtures with a poor solvent, DMSO, in order to generate pores on PCL fiber surface. The production of porous, bead free fibers was achieved using $12.5 \% \mathrm{w} / \mathrm{v}$ PCL in CF/DMSO solution with good/poor solvent ratios varying from $75 \%$ to $90 \% \mathrm{v} / \mathrm{v}$. However, DCM and THF were proven to be less suitable good solvents for the process due to the formation of a solid skin on the jet surface, caused by the limited diffusivity of the polymer molecules from the jet surface to the liquid core and its subsequent collapse. FA was found to be unsuitable due to its similar evaporation rate to DMSO. The production of fibers with ribbon cross sections or fibers with beads was more pronounced at low good/poor solvent ratios. In another investigation, reproducible buckled and porous sub-micron diameter electrospun PCL fibers were produced by simple electrospinning process for biomedical applications (Luwang Laiva et al., 2014). PCL solutions were prepared with in the binary solvent combinations (with different vapor pressures) of CF with ethanol, DCM, DMF, DMSO, ethyl acetate and diethyl ether a ratio of 9:1 v/v respectively, to make a 16\% (w/v) solution for electrospinning. Fiber morphologies was affected with various degree by solvent combinations used for the fabrication of sub-micron fibers. The results suggested that the solution viscosity, the collecting distance and the type of solvent combination used could be an optimum parameter for the generation of porous-buckled fibers with narrow pore size distribution. Georgiadou et al. (Katsogiannis, Vladisavljević, and Georgiadou, 2016) also confirmed that electrospinning process parameters had an effect on surface morphology of porous electrospun fibers which was obtained by using PCL solution in a binary solvent mixture of 90/10 \% v/v CF/ DMSO. In another study, PAN porous fibers were spun in one step by electrospinning a ternary system of PAN/DMF/water (X. Yu et al., 2010). The spinodal decomposition phase separation resulted in the porous structure. Fortunato et al. (Yazgan et al., 2017) confirmed that irregular and interconnected surface topographies was obtained on PCL fibers by using CF/DMSO solvent system beginning of 35\% RH. Megelski et al. (Megelski, Stephens, Chase, and Rabolt, 2002) observed that the ratio of the less volatile DMF increased in THF/DMF solvent mixtures, therefore reducing the vapor pressure, surface roughness or microtexture was observed of PS fibers. The microtexture finally disappeared, leaving a smooth surface with decreasing solvent volatility by using $100 \%$ DMF.

\subsection{Vapor induced phase separation (VIPS)}

In the VIPS method, vapors (usually water) are condensed as nonsolvents that cause originally homogeneous solutions to phase separate and porous structures can be obtained after the removal of solvents (C.-L. Li et al., 2010; Srinivasarao et al., 2001). VIPS also occurs in the electrospinning process (Figure $1 \mathrm{c}$ ). Solvent evaporation cools down the jet surface and the moisture condense from the atmosphere. Initiating of the nucleation and growth of the water-rich phase floating on the solution subsequently leaves pores on the fiber surface (Lu and Xia, 2013; Megelski et al., 2002; Nezarati et al., 2013; Park and Lee, 2010; Wu et al., 2012).

Many researches highlighted that the effects of relative humidity on electrospun fiber morphology are dependent on polymer hydrophobicity, solvent miscibility with water, and solvent volatility (Nezarati et al., 2013). Three polymers (poly(ethylene 
glycol) (PEG), PCL, and poly(carbonate urethane) (PCU) were electrospun at a range of relative humidities $(\mathrm{RH}=5 \%-75 \%)$. At high relative humidity $(>50 \%)$, three distinct effects were observed based on individual polymer properties. An increase in fiber breakage and loss of fiber morphology occurred in the PEG system as a result of increased water absorption at high relative humidity. In contrast, surface pores on PCL fibers were observed and hypothesized to have formed via VIPS. Finally, decreased PCU fiber collection occurred at high humidity likely due to increased electrostatic discharge. It was demonstrated that the amount of moisture in the air affected the surface morphology of electrospun PS fibers from THF (Casper et al., 2004). Electrospinning in an atmosphere of less than $25 \%$ humidity produced smooth fibers without any surface features. When the humidity was above $30 \%$, pores began to form on the surface of the fiber. Increasing the amount of humidity caused an increase in the number of pores on the surface, the pore diameter, and the pore size distribution. In addition to that higher molecular weight solutions caused fibers to contain larger pores that were less uniform in shape and size. Three solvent systems (i.e., DMF, THF, and DMF - THF mixture) were tested under four controlled levels of relative humidity (Lu and Xia, 2013). Whereas only solid PS fibers with smooth surfaces were produced at low relative humidity (2\%), both internally and externally porous PS fibers/yarns were obtained by conducting electrospinning at high relative humidity $(22 \%, 42 \%$, and $62 \%)$. Furthermore, it was discovered that the low vapor pressure of DMF facilitated the formation of internal porosity while the high vapor pressure of THF hindered the formation of interior pores and could only produce surface porosity. Megelski et al. (Megelski et al., 2002) proved that increasing relative humidity from 20 to $50 \%$ would increase the pore formation for the fibers from PS/THF polymer/solvent system.

\subsection{Thermally induced phase separation (TIPS)}

In the TIPS method, a homogeneous polymer solution is first prepared at an elevated temperature and then rapidly cooled to a temperature where the solvent quality turn to be poor to polymer and phase separation occurs (Chang, Beltsios, Chen, Lin, and Cheng, 2014). This method can be applied to produce electrospinning porous polymer fibers by controlling the collector at relatively low temperature to induce phase separation (McCann, Marquez, and Xia, 2006). This method needs post-treatments to remove residual solvents because the fibers are not completely dried in the electrospinning process.

McCann et al.(McCann et al., 2006) exploited the role of TIPS in the creation of highly porous electrospun nanofibers by using a liquid nitrogen bath as the quenching medium. The fibers were frozen in the liquid nitrogen bath resulting in TIPS. It was noted that the distance between the metallic needle and the collector should be chosen properly to ensure that the solvent could not be completely evaporated before the jet reached the liquid nitrogen. Lee et al. (J. F. Kim, Kim, Lee, and Drioli, 2016) reviewed TIPS and electrospinning methods for preparation of fluoropolymer membranes, particularly for the polyvinylidene fluoride and polyethylene chlorotrifluoroethylene membranes. They focused on controlling the membrane morphology from the thermodynamic and kinetic perspectives to understand the relationship between the membrane morphology and fabrication parameters. It was reported that by immersing the collector in a bath of liquid nitrogen, porous PS fibers can be obtained through TIPS between the solvent-rich and solvent-poor regions in the fiber during electrospinning (McCann et al., 2006). Isotactic polypropylene and poly(vinylidene fluoride) porous fibers were successfully prepared as two of the most commonly used membrane materials by electrospinning at $200{ }^{\circ} \mathrm{C}$ combined with TIPS (Ye, Lin, Huang, Liang, and Xu, 2013). The as-spun porous fibers have high porosity and more than a 100-fold increase in specific surface area compared with non-porous fibers, which would dramatically improve their in-service performance as separation media.

\subsection{Another approaches}

Porous nanofibers could be prepared by the selective removal of a component from nanofibers made of composite or blended materials. For example, the structural changes for fibers consisting of a PLA/polyvinylpyrrolidone (PVP) blend were investigated when one of the two components was selectively removed from the composites (Bognitzki et al., 2001). It was found that porous nanofibers were obtained after selective removal of PVP by water extraction or alternatively to remove PLA by annealing at elevated temperatures, when equal amount of the two polymers were loaded into the electrospinning solution. A leaching treatment from bi-continuous material of gelatin/PCL nanofibers lead to the formation of novel 3D porous nanofibers with reduced fiber diameter (Zhang, Feng, Huang, Ramakrishna, and Lim, 2006). The fiber surface after leaching treatment became rough and irregular because of the existence of many interconnected striated ridges, grooves and ellipsoidal shaped pores with the long axis oriented in the direction of the fiber axis. Gupta et al. (Gupta et al., 2009) produced porous nylon-6 fibers from the Lewis acid base complication of gallium trichloride $(\mathrm{GaCl} 3)$ and nylon- 6 using electrospinning, followed by $\mathrm{GaCl} 3$ removal. In another study, highly porous fibers were prepared by water-bath electrospinning from PCL, and its blends with methoxy poly(ethylene glycol) (MPEG) as potential tissue scaffold material (Pant et al., 2011). A new approach was reported to fabricate electrospun polymer nonwoven mats with porous surface morphology by varying the collector temperature during electrospinning (C. H. Kim et al., 2006). Polymers such as poly(L-lactide), PS, and poly(vinyl acetate) were dissolved in volatile solvents, DCM and THF, and subjected to electrospinning. The temperature of the collector in the electrospinning device was varied by a heating system. According to the results the surface morphology, porous structure, and the properties such as pore size, depth, shape, and distribution of the nonwoven mats were greatly influenced by the collector temperature. In a 
study, inter-surface-connected porous fibers based on rapid phase separation between poly(propylene carbonate) and PCL were produced (Wang et al., 2015). ADCs cultured on electrospun fibers with intersurface-connected pores showed increased cellular adhesion, proliferation and differentiation than those cultured on the fiber with blind-holes. In another study, the morphology, structure and tensile properties of PLA porous nanofibers were studied (Y. Li, Lim, and Kotaki, 2015). Different crystallization ability of PLA was electrospun from mixed solvent of DCM and DMF into porous nanofibers through two kinds of spinnerets (nozzle and channel spinnerets) in a highly humid environment. Results showed that take-up velocity affected the porous morphology of nanofibers, which was more obvious in the case of channel-based spinneret and PLA with low crystallization ability. In the case of PLA with high crystallization ability, an increase in take-up velocity led to more highly packed internal structure, which could contribute to the enhancement in tensile properties. In addition, tensile properties of porous nanofibers of PLA with low crystallization ability could be manipulated by surface morphology control via channel-based electrospinning system.

Many researchers have also verified that electrospinning parameters, including molecular weight of polymer (Casper et al., 2004), polymer concentration (Kongkhlang et al., 2008; J. Lin et al., 2010), solvent (Kongkhlang et al., 2008; J. Lin et al., 2010), spinning voltage (Kongkhlang et al., 2008), had an influence on morphology of porous nanofiber in terms of nanoporosity as well as size, shape, and distribution of these pores.

\section{CONCLUSION}

Porous materials have found widespread use in a wide variety of applications such as filtration, catalysis, and biomedical research. Through rational design and controlled fabrication, it is possible to obtain desired porous fibers at different characteristics. Since different mechanisms resulting from relative humidity, solvent and polymer properties and also electrospinning conditions involves in the processing, it essential to carefully design experimental setup for various applications.

\section{REFERENCES}

Baker, B. M., Trappmann, B., Wang, W. Y., Sakar, M. S., Kim, I. L., Shenoy, V. B., . . Chen, C. S. (2015). Cell-mediated fibre recruitment drives extracellular matrix mechanosensing in engineered fibrillar microenvironments. Nature materials, 14(12), $1262-1268$.

Bognitzki, M., Frese, T., Steinhart, M., Greiner, A., Wendorff, J. H., Schaper, A., and Hellwig, M. (2001). Preparation of fibers with nanoscaled morphologies: Electrospinning of polymer blends. Polymer Engineering and Science, 41(6), 982-989.

Casper, C. L., Stephens, J. S., Tassi, N. G., Chase, D. B., and Rabolt, J. F. (2004). Controlling Surface Morphology of Electrospun Polystyrene Fibers: Effect of Humidity and Molecular Weight in the Electrospinning Process. Macromolecules, 37(2), 573-578.

Chang, H.-H., Beltsios, K., Chen, Y.-H., Lin, D.-J., and Cheng, L.-P. (2014). Effects of cooling temperature and aging treatment on the morphology of nano- and micro-porous poly(ethylene-co-vinyl alcohol) membranes by thermal induced phase separation method. Journal of Applied Polymer Science, 131(12).

Chen, P.-Y., and Tung, S.-H. (2017). One-Step Electrospinning To Produce Nonsolvent-Induced Macroporous Fibers with Ultrahigh Oil Adsorption Capability. Macromolecules, 50(6), 2528-2534.

Gupta, A., Saquing, C. D., Afshari, M., Tonelli, A. E., Khan, S. A., and Kotek, R. (2009). Porous Nylon-6 Fibers via a Novel Salt-Induced Electrospinning Method. Macromolecules, 42(3), 709-715.

Katsogiannis, K. A. G., Vladisavljević, G. T., and Georgiadou, S. (2015). Porous electrospun polycaprolactone (PCL) fibres by phase separation. European Polymer Journal, 69, 284-295.

Katsogiannis, K. A. G., Vladisavljević, G. T., and Georgiadou, S. (2016). Porous electrospun polycaprolactone fibers: Effect of process parameters. Journal of Polymer Science Part B: Polymer Physics, 54(18), 1878-1888.

Kim, C. H., Jung, Y. H., Kim, H. Y., Lee, D. R., Dharmaraj, N., and Choi, K. E. J. M. R. (2006). Effect of collector temperature on the porous structure of electrospun fibers. Macromolecular Research, 14(1), 59-65.

Kim, J. F., Kim, J. H., Lee, Y. M., and Drioli, E. (2016). Thermally induced phase separation and electrospinning methods for 
emerging membrane applications: A review. Advances in Materials. Separations: Materials, Devices and Processes, 62(2), 461-490.

Kongkhlang, T., Kotaki, M., Kousaka, Y., Umemura, T., Nakaya, D., and Chirachanchai, S. (2008). Electrospun Polyoxymethylene: Spinning Conditions and Its Consequent Nanoporous Nanofiber. Macromolecules, 41(13), 4746-4752.

Li, C.-L., Wang, D.-M., Deratani, A., Quémener, D., Bouyer, D., and Lai, J.-Y. (2010). Insight into the preparation of poly(vinylidene fluoride) membranes by vapor-induced phase separation. Journal of Membrane Science, 361(1), 154-166.

Li, D., and Xia, Y. (2004). Electrospinning of Nanofibers: Reinventing the Wheel? Advanced Materials, 16(14), 11511170.

Li, Y., Lim, C. T., and Kotaki, M. (2015). Study on structural and mechanical properties of porous PLA nanofibers electrospun by channel-based electrospinning system. Polymer, 56, 572-580.

Lin, D.-J., Chang, H.-H., Chen, T.-C., Lee, Y.-C., and Cheng, L.-P. (2006). Formation of porous poly(vinylidene fluoride) membranes with symmetric or asymmetric morphology by immersion precipitation in the water/TEP/PVDF system. European Polymer Journal, 42(7), 1581-1594.

Lin, J., Ding, B., and Yu, J. (2010). Direct fabrication of highly nanoporous polystyrene fibers via electrospinning. ACS Appl Mater Interfaces, 2(2), 521-528.

Liu, C., Hsu, P.-C., Lee, H.-W., Ye, M., Zheng, G., Liu, N., . . Cui, Y. (2015). Transparent air filter for high-efficiency PM2.5 capture. Nature Communications, 6, 6205.

Lu, P., and Xia, Y. (2013). Maneuvering the internal porosity and surface morphology of electrospun polystyrene yarns by controlling the solvent and relative humidity. Langmuir, 29(23), 7070-7078.

Lubasova, D., and Martinova, L. (2011). Controlled Morphology of Porous Polyvinyl Butyral Nanofibers \%J Journal of Nanomaterials. 2011, 6 .

Luwang Laiva, A., Venugopal, J. R., Sridhar, S., Rangarajan, B., Navaneethan, B., and Ramakrishna, S. (2014). Novel and simple methodology to fabricate porous and buckled fibrous structures for biomedical applications. Polymer, 55(22), 5837-5842.

Marcos-Martin, M., Beysens, D., Bouchaud, J. P., Godrèche, C., and Yekutieli, I. (1995). Self-diffusion and 'visited' surface in the droplet condensation problem (breath figures). Physica A: Statistical Mechanics and its Applications, 214(3), 396-412.

McCann, J. T., Marquez, M., and Xia, Y. (2006). Highly Porous Fibers by Electrospinning into a Cryogenic Liquid. Journal of the American Chemical Society, 128(5), 1436-1437.

Megelski, S., Stephens, J. S., Chase, D. B., and Rabolt, J. F. (2002). Micro- and Nanostructured Surface Morphology on Electrospun Polymer Fibers. Macromolecules, 35(22), 8456-8466.

Nayani, K., Katepalli, H., Sharma, C. S., Sharma, A., Patil, S., and Venkataraghavan, R. (2012). Electrospinning Combined with Nonsolvent-Induced Phase Separation To Fabricate Highly Porous and Hollow Submicrometer Polymer Fibers. Industrial and Engineering Chemistry Research, 51(4), 1761-1766.

Nezarati, R. M., Eifert, M. B., and Cosgriff-Hernandez, E. (2013). Effects of humidity and solution viscosity on electrospun fiber morphology. Tissue Engineering Part C Methods, 19(10), 810-819.

Nguyen, T.-H., Bao, T. Q., Park, I., and Lee, B.-T. (2013). A novel fibrous scaffold composed of electrospun porous poly( $\varepsilon$-caprolactone) fibers for bone tissue engineering. Journal of Biomaterials Applications, 28(4), 514-528. 
Pant, H. R., Neupane, M. P., Pant, B., Panthi, G., Oh, H. J., Lee, M. H., and Kim, H. Y. (2011). Fabrication of highly porous poly (varepsilon-caprolactone) fibers for novel tissue scaffold via water-bath electrospinning. Colloids and Surfaces B: Biointerfaces, 88(2), 587-592.

Park, J. Y., and Lee, I. H. (2010). Relative humidity effect on the preparation of porous electrospun polystyrene fibers. J Nanosci Nanotechnol, 10(5), 3473-3477.

Qi, Z., Yu, H., Chen, Y., and Zhu, M. (2009). Highly porous fibers prepared by electrospinning a ternary system of nonsolvent/solvent/poly(l-lactic acid). Materials Letters, 63(3), 415-418.

Ramakrishna, S. A. F., Kazutoshi\%A Teo, Wee-Eong\%A Lim, Teik-Cheng\%A Ma, Zuwei. An Introduction to Electrospinning and Nanofibers.

Srinivasarao, M., Collings, D., Philips, A., and Patel, S. (2001). Three-Dimensionally Ordered Array of Air Bubbles in a Polymer Film. Science, 292(5514), 79-83.

Şimşek, M. (2018). Fabrication of Fibrous Tissue Scaffolds. Dubai: GlobeEdit.

Wang, Y., Deng, J., Fan, R., Tong, A., Zhang, X., Zhou, L., . . . Guo, G. (2015). Novel nanoscale topography on poly(propylene carbonate)/poly( $\varepsilon$-caprolactone) electrospun nanofibers modifies osteogenic capacity of ADCs. RSC Advances, 5(101), 82834-82844.

Wei, Z., Zhang, Q., Wang, L., Wang, X., Long, S., Yang, J. J. C., and Science, P. (2013). Porous electrospun ultrafine fibers via a liquid-liquid phase separation method. Colloid and Polymer Science, 291(5), 1293-1296.

Wu, J., Wang, N., Wang, L., Dong, H., Zhao, Y., and Jiang, L. (2012). Electrospun Porous Structure Fibrous Film with High Oil Adsorption Capacity. ACS Appl Mater Interfaces, 4(6), 3207-3212.

Yazgan, G., Dmitriev, R. I., Tyagi, V., Jenkins, J., Rotaru, G.-M., Rottmar, M., . . . Fortunato, G. (2017). Steering surface topographies of electrospun fibers: understanding the mechanisms. Scientific Reports, 7(1), 158.

Ye, X.-Y., Lin, F.-W., Huang, X.-J., Liang, H.-Q., and Xu, Z.-K. (2013). Polymer fibers with hierarchically porous structure: combination of high temperature electrospinning and thermally induced phase separation. RSC Advances, 3(33), 13851-13858.

Yu, J., Qiu, Y., Zha, X., Yu, M., Yu, J., Rafique, J., and Yin, J. (2008). Production of aligned helical polymer nanofibers by electrospinning. European Polymer Journal, 44(9), 2838-2844.

Yu, X., Xiang, H., Long, Y., Zhao, N., Zhang, X., and Xu, J. (2010). Preparation of porous polyacrylonitrile fibers by electrospinning a ternary system of PAN/DMF/H2O. Materials Letters, 64(22), 2407-2409.

Zhang, Y. Z., Feng, Y., Huang, Z. M., Ramakrishna, S., and Lim, C. T. (2006). Fabrication of porous electrospun nanofibres. Nanotechnology, 17(3), 901-908. 